\title{
Detection and discrimination of cosmological non-Gaussian signatures by multi-scale methods ${ }^{\star}$
}

\author{
J.-L. Starck ${ }^{1}$, N. Aghanim ${ }^{2}$, and O. Forni ${ }^{2}$ \\ 1 DAPNIA/SEDI-SAP, Service d'Astrophysique, CEA-Saclay, 91191 Gif-sur-Yvette Cedex, France \\ 2 IAS-CNRS, Université Paris Sud, Bâtiment 121, 91405, Orsay Cedex, France
}

Received 19 February 2003 / Accepted 6 November 2003

\begin{abstract}
Recent Cosmic Microwave Background (CMB) observations indicate that the temperature anisotropies arise from quantum fluctuations in the inflationary scenario. In the simplest inflationary models, the distribution of CMB temperature fluctuations should be Gaussian. However, non-Gaussian signatures can be present. They might have different origins and thus different statistical and morphological characteristics.

In this context and motivated by recent and future CMB experiments, we search for, and discriminate between, different non-Gaussian signatures. We analyse simulated maps of three cosmological sources of temperature anisotropies: Gaussian distributed $\mathrm{CMB}$ anisotropies from inflation, temperature fluctuations from cosmic strings and anisotropies due to the kinetic Sunyaev-Zel'dovich (SZ) effect both showing a non-Gaussian character. We use different multi-scale methods, namely, wavelet, ridgelet and curvelet transforms. The sensitivity and the discriminating power of the methods is evaluated using simulated data sets.

We find that the bi-orthogonal wavelet transform is the most powerful for the detection of non-Gaussian signatures and that the curvelet and ridgelet transforms characterise quite precisely and exclusively the cosmic strings. They allow us thus to detect them in a mixture of $\mathrm{CMB}+\mathrm{SZ}+$ cosmic strings. We show that not one method only should be applied to understand non-Gaussianity but rather a set of different robust and complementary methods should be used.
\end{abstract}

Key words. cosmology: cosmic microwave background - cosmology: early universe - methods: data analysis

\section{Introduction}

The search for non-Gaussian signatures in the Cosmic Microwave Background (CMB) temperature fluctuation maps furnished by MAP $^{1}$ (Komatsu et al. 2003), and to be furnished by PLANCK $^{2}$, is of great interest for cosmologists. Indeed, the non-Gaussian signatures in the CMB can be related to very fundamental questions such as the global topology of the universe (Riazuelo et al. 2002), super string theory, topological defects such as cosmic strings (Bouchet et al. 1988), and multi-field inflation (Bernardeau \& Uzan 2002). The non-Gaussian signatures can, however, have a different but still cosmological origin. They can be associated with the Sunyaev-Zel'dovich (SZ) effect (Sunyaev \& Zeldovich 1980) (inverse Compton effect) of the hot and ionised intra-cluster gas of galaxy clusters (Aghanim \& Forni 1999; Cooray 2001), with the gravitational lensing by large scale structures (Bernardeau et al. 2003), or with the reionisation of the universe (Aghanim \& Forni 1999;

\footnotetext{
Send offprint requests to: J.-L. Starck, e-mail: jstarck@cea.fr

* Appendix A is only available in electronic form at

http://www.edpsciences.org

${ }^{1}$ http://map.gsfc.nasa.gov/

2 http://astro.estec.esa.nl/SA-general/Projects/ Planck/
}

Castro 2003). They may also be simply due to foreground emission (Jewell 2001), or to non-Gaussian instrumental noise and systematics (Banday et al. 2000).

It has now become clear that the detection of non-Gaussian signature is important and feasible (e.g. De Troia et al. 2003; Komatsu et al. 2003). Nevertheless, even if a non-Gaussian signal is detected the question of its origin might still be posed (e.g. Banday et al. 2000). Hence, it remains to separate between several non-Gaussian contributions. This is the most crucial issue for the use the non-Gaussian signatures as cosmological tools.

It is therefore not surprising that a large number of studies have recently been devoted to the subject of the detection of non-Gaussian signatures. Many approaches have been investigated such as the Minkowski functionals and the morphological statistics (Novikov et al. 2000; Shandarin 2002), the bispectrum (Bromley \& Tegmark 1999; Verde et al. 2000; Phillips $\&$ Kogut 2001), the trispectrum (Kunz et al. 2001), or wavelet transforms (Aghanim \& Forni 1999; Forni \& Aghanim 1999; Hobson et al. 1999; Barreiro \& Hobson 2001; Cayón et al. 2001; Jewell 2001). Different wavelet methods have been studied, such as the à trous algorithm (Starck et al. 1998) and the bi-orthogonal wavelet transform (Mallat 1998). 
A series of recent papers (Candès \& Donoho 1999a,b; Starck et al. 2002), however, argued that wavelets and related classical multi-resolution techniques are based on a limited dictionary made up of roughly isotropic elements present at all scales and locations. We view as a limitation the fact that those dictionaries do not exhibit highly anisotropic elements and that there is only a fixed number of directional elements, independent of scale. Despite the success of the classical wavelet viewpoint, there are objects, such as filamentary structures or cosmic strings, that do not exhibit isotropic scaling and thus call for other kinds of multi-scale representations. Following this, new multi-scale systems like curvelets and ridgelets (Candès \& Donoho 1999b) have been introduced which are very different from wavelet-like systems. Curvelets and ridgelets take the form of basis elements which exhibit very high directional sensitivity and are highly anisotropic. In two dimensions, for instance, curvelets are localised along curves, in three dimensions along sheets, etc.

The goal of the present study is to compare these new multi-scale representations to standard wavelet methods on a set of simulated maps representing two generic families of nonGaussianities (spherical-like and string-like sources), and to answer the following questions:

1. Are there multi-scale methods that are better suited to detect the non-Gaussian signatures in CMB data?

2. Can we go beyond the detection and extract some information about the nature of the non-Gaussian signal?

The second point is particularly important and it will represent the heart of our present study. A non-Gaussian character can be due to many effects: topological defects, the SZ effect, calibration problems, or even a combination of them. Here, we perform a study based on the idea that a given multi-scale transform is optimal to detect features which have the shape of its basis elements (this is quite similar to the matched filter approach). Consequently, if different multi-scale methods are able to detect a non-Gaussian signal, one of them will outperform the others since the shape of the non-Gaussian features contained in the data will be close to its basis elements. In particular, using wavelet-like systems and curvelet and ridgelet-like systems, we expect to differentiate between the spherical-like and string-like sources of non-Gaussianity.

Sections 2 and 3 briefly review the different transforms and simulated maps considered in our analysis. We present the analysis together with our main results in Sect. 4 and discuss them in Sect. 5. We provide the main conclusions in Sect. 6.

\section{Multi-scale transforms}

\subsection{Bi-orthogonal wavelet transforms}

The most commonly used wavelet transform algorithm is the decimated bi-orthogonal wavelet transform (OWT). Using the OWT, a signal $s$ can be decomposed as follows:

$$
s(l)=\sum_{k} c_{J, k} \phi_{J, l}(k)+\sum_{k} \sum_{j=1}^{J} \psi_{j, l}(k) w_{j, k}
$$

with $\phi_{j, l}(x)=2^{-j} \phi\left(2^{-j} x-l\right)$ and $\psi_{j, l}(x)=2^{-j} \psi\left(2^{-j} x-l\right)$, where $\phi$ and $\psi$ are respectively the scaling and the wavelet functions. $J$ is the number of resolutions used in the decomposition, $w_{j}$ the wavelet coefficients (or details) at scale $j$, and $c_{J}$ is a coarse or smooth version of the original signal $s$. The present indexing is such that $j=1$ corresponds to the finest scale (high frequencies).

The two-dimensional algorithm is based on separate variables leading to prioritising of horizontal, vertical and diagonal directions. The scaling function is defined by $\phi(x, y)=$ $\phi(x) \phi(y)$, and the detail signal is obtained from three wavelets:

$$
\begin{aligned}
& \text { - vertical wavelet: } \psi^{1}(x, y)=\phi(x) \psi(y) \\
& \text { - horizontal wavelet: } \psi^{2}(x, y)=\psi(x) \phi(y) \\
& \text { - diagonal wavelet: } \psi^{3}(x, y)=\psi(x) \psi(y)
\end{aligned}
$$

which leads to three wavelet sub-images at each resolution level. A given wavelet band is therefore defined by its resolution level $j(j=1 \ldots J)$ and its direction number $d(d=1 \ldots 3$, corresponding respectively to the horizontal, vertical, and diagonal band).

The OWT presents only a fixed number of directional elements independent of scales, and there are no highly anisotropic elements (Candès \& Donoho 1999a). For instance, the Haar 2D wavelet transform is optimal to find features with a ratio length/width $=2$, and a horizontal, vertical, or diagonal orientation. Therefore, we naively expect the OWT to be optimal for detecting mildly isotropic or anisotropic features.

\subsection{The isotropic à trous wavelet transform}

The à trous wavelet transform algorithm decomposes an $n \times n$ image $I$ as a superposition of the form

$I(x, y)=c_{J}(x, y)+\sum_{j=1}^{J} w_{j}(x, y)$,

where $c_{J}$ is a coarse or smooth version of the original image $I$ and $w_{j}$ represents "the details of $I$ " at scale $2^{-j}$ (see Starck et al. (1998, 2002) for more information). Thus, the algorithm outputs $J+1$ sub-band arrays of size $n \times n$. (The present indexing is such that $j=1$ corresponds to the finest scale (high frequen(ies).)

Hence, we have a multi-scale pixel representation, i.e. each pixel of the input image is associated with a set of pixels of the multi-scale transform. This wavelet transform is very well adapted to the detection of isotropic features, and this explains its success for astronomical image processing, where the data contain mostly isotropic or quasi-isotropic objects, such as stars, galaxies or galaxy clusters.

\subsection{The ridgelet transform}

The two-dimensional continuous ridgelet transform in $\mathbf{R}^{2}$ can be defined as follows (Candès \& Donoho 1999a). We pick a smooth univariate function $\psi: \mathbf{R} \rightarrow \mathbf{R}$ with sufficient decay and satisfying the admissibility condition

$\int|\hat{\psi}(\xi)|^{2} /|\xi|^{2} \mathrm{~d} \xi<\infty$ 
which holds if, say, $\psi$ has a vanishing mean $\int \psi(t) \mathrm{d} t=0$. We will suppose that $\psi$ is normalised so that $\int|\hat{\psi}(\xi)|^{2} \xi^{-2} \mathrm{~d} \xi=1$.

For each $a>0$, each $b \in \mathbf{R}$ and each $\theta \in[0,2 \pi]$, we define the bivariate ridgelet $\psi_{a, b, \theta}: \mathbf{R}^{2} \rightarrow \mathbf{R}$ by

$\psi_{a, b, \theta}(\boldsymbol{x})=a^{-1 / 2} \cdot \psi\left(\left(x_{1} \cos \theta+x_{2} \sin \theta-b\right) / a\right)$.

Given an integrable bivariate function $f(\boldsymbol{x})$, we define its ridgelet coefficients by:

$\mathcal{R}_{f}(a, b, \theta)=\int \bar{\psi}_{a, b, \theta}(\boldsymbol{x}) f(\boldsymbol{x}) \mathrm{d} \boldsymbol{x}$.

We have the exact reconstruction formula

$f(\boldsymbol{x})=\int_{0}^{2 \pi} \int_{-\infty}^{\infty} \int_{0}^{\infty} \mathcal{R}_{f}(a, b, \theta) \psi_{a, b, \theta}(\boldsymbol{x}) \frac{\mathrm{d} a}{a^{3}} \mathrm{~d} b \frac{\mathrm{d} \theta}{4 \pi}$

valid for functions which are both integrable and square integrable.

It has been shown (Candès \& Donoho 1999a) that the ridgelet transform is precisely the application of a 1-dimensional wavelet transform to the slices of the Radon transform.

\section{Local ridgelet transform}

The ridgelet transform is optimal to find only lines of the size of the image. To detect line segments, a partitioning must be introduced. The image is decomposed into smoothly overlapping blocks of side-length $B$ pixels in such a way that the overlap between two vertically adjacent blocks is a rectangular array of size $B \times B / 2$; we use an overlap to avoid blocking artifacts. For an $n \times n$ image, we count $2 n / B$ such blocks in each direction. The partitioning introduces redundancy, as a pixel belongs to 4 neighboring blocks.

More details on the implementation of the digital ridgelet transform can be found in Starck et al. $(2002,2003)$. The ridgelet transform is therefore optimal to detect lines of a given size, which is the block size.

\subsection{The curvelet transform}

The curvelet transform (Donoho \& Duncan 2000; Starck et al. 2003) opens the possibility to analyse an image with different block sizes, but with a single transform. The idea is to first decompose the image into a set of wavelet bands, and to analyse each band with a local ridgelet transform. The block size can be changed at each scale level. Roughly speaking, different levels of the multi-scale ridgelet pyramid are used to represent different sub-bands of a filter bank output.

The side-length of the localising windows is doubled at every other dyadic sub-band, hence maintaining the fundamental property of the curvelet transform, that elements of length about $2^{-j / 2}$ serve for the analysis and synthesis of the $j$ th subband $\left[2^{j}, 2^{j+1}\right]$. Note also that the coarse description of the image $c_{J}$ is not processed. In our implementation, we used the default block size value $B_{\min }=16$ pixels. This implementation of the curvelet transform is also redundant. The redundancy factor is equal to $16 J+1$ whenever $J$ scales are employed.
A given curvelet band is therefore defined by the resolution level $j(j=1 \ldots J)$ related to the wavelet transform, and by the ridgelet scale $r$.

This method is optimal to detect anisotropic structures of different lengths.

\subsection{Statistics from the multi-scale coefficients}

Many kinds of statistics can be derived from the multi-scale coefficients (Aghanim \& Forni 1999). In the following we use the kurtosis of the coefficients obtained by the previously described multi-scale transforms. Several aspects have however to be considered.

\section{The border problem}

Wavelet coefficients have been obtained by convolution with filters, and coefficients close to the border of the image have therefore been calculated using an extrapolation and should not be used when calculating the excess kurtosis. For the biorthogonal wavelet transform, we use the 7/9 filter, which implies that coefficients at a distance from the border smaller than three must not be taken into account. For à trous wavelet coefficients at scale $j$, the distance is $2^{j}$ (because it is an undecimated wavelet transform). For the same reasons, border blocks in the ridgelet and the curvelet transforms must also not be used.

\section{Renormalisation of the ridgelet and the curvelet coefficients}

When using the ridgelet and the curvelet transforms, another problem occurs. Indeed, as a coefficient is obtained by integrating along a given direction, the expectation value of a coefficient depends on both the direction and the position of the line. For example, coefficients relative to diagonal directions integrate more values than those relative to horizontal and vertical directions. A renormalisation is needed. After applying the ridgelet transform independently to all blocks, we obtained a set of $N_{\mathrm{t}}$ transform blocks $T_{i}(a, b, \theta)(i=$ $\left.1 \ldots N_{\mathrm{t}}\right)$, and for each scale, orientation and position $(a, b, \theta)$, we extract the vector $V_{a, b, \theta}(i)$. For a stationary signal, dividing $T_{i}(a, b, \theta)$ by the standard deviation of $V_{a, b, \theta}$ leads to a good renormalisation, but it is not our case, (cosmological signals are not stationary) and we therefore use a robust estimator, MAD (Median Absolute Deviation), defined by $\operatorname{MAD}(x)=$ median $(|x|) / 0.6745$ (Rousseeuw \& Croux 1993). Hence, we normalise the ridgelet coefficients by the following expression:

$\bar{T}_{i}(a, b, \theta)=\frac{T_{i}(a, b, \theta)}{\operatorname{MAD}\left(V_{a, b, \theta}\right)}$.

For the curvelet transform, this renormalisation is performed independently on each scale.

\section{Simulated astrophysical signals}

The temperature anisotropies of the CMB contain the contributions of both the primary cosmological signal, directly related to the initial density perturbations, and the secondary 


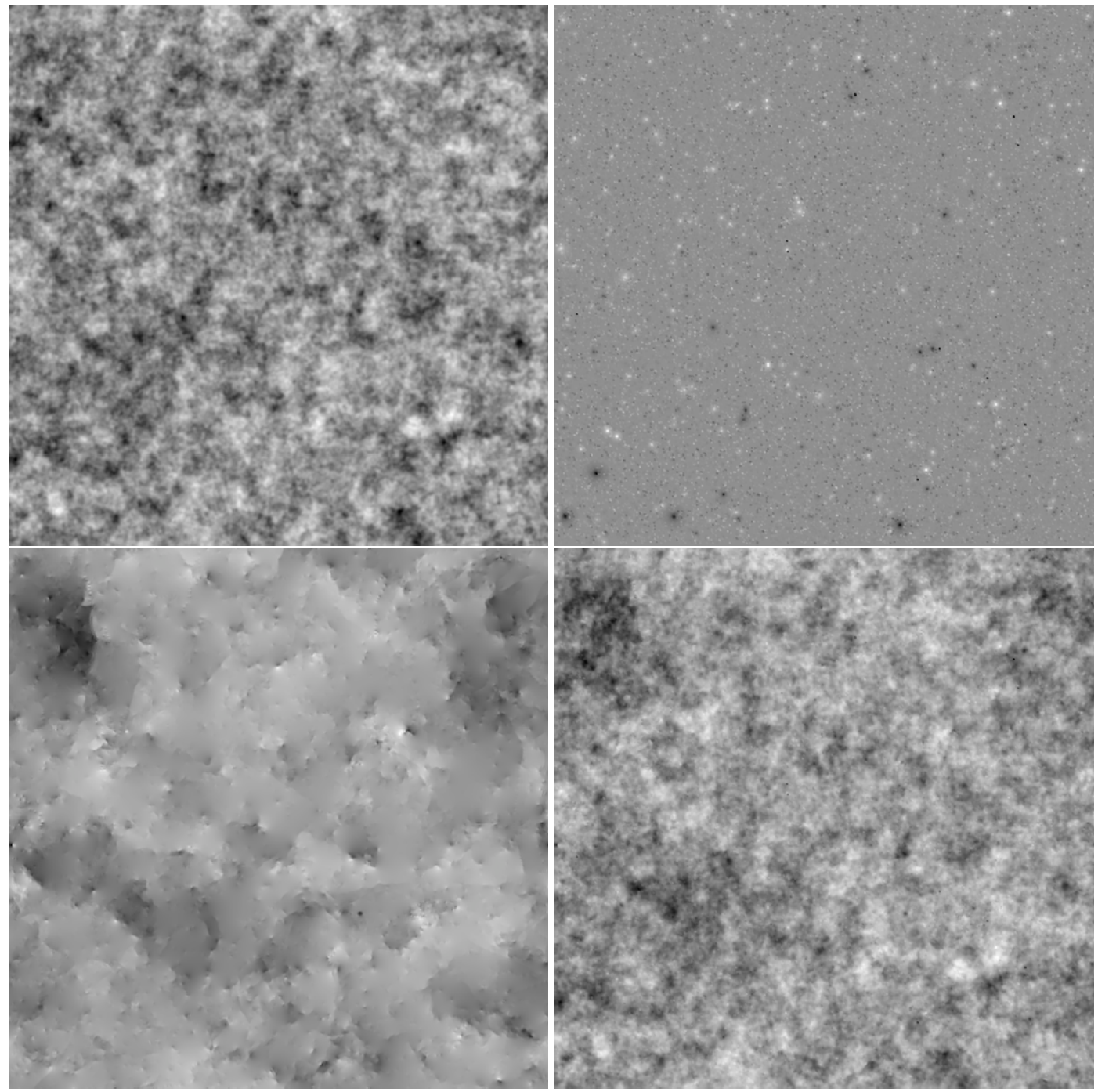

Fig. 1. Top, primary Cosmic Microwave Background anisotropies (left) and kinetic Sunyaev-Zel'dovich fluctuations (right). Bottom, cosmic string simulated map (left) and simulated observation containing the previous three components (right).

anisotropies. The latter are generated after matter-radiation decoupling (White \& Cohn 2002). They arise from the interaction of the CMB photons with the neutral or ionised matter along their path (Sunyaev \& Zeldovich 1980; Ostriker \& Vishniac 1986; Vishniac 1987).

In the present study, we assume that the primary CMB anisotropies are dominated by the fluctuations generated in the simple single field inflationary Cold Dark Matter model with a non-zero cosmological constant. The CMB anisotropies have therefore a Gaussian distribution. We allow for a contribution to the primary signal from topological defects, namely cosmic strings (CS), as suggested in (Bouchet et al. 2002). In addition, we take into account the secondary anisotropies due to the kinetic Sunyaev-Zel'dovich (SZ) effect (Sunyaev $\&$ Zeldovich 1980). The SZ effect represents the Compton scattering of the CMB photons by the free electrons of the ionised and hot intra-cluster gas. When the galaxy cluster moves with respect to the CMB rest frame, the Doppler shift induces additional anisotropies; this is the so-called kinetic SZ (KSZ) effect. As the latter fluctuations have the same spectral signature as the primary, we add the two signals directly. The kinetic SZ maps are simulated following Aghanim et al. (2001). We use for our simulations the cosmological parameters obtained from the WMAP satellite (Bennett et al. 2003) and a normalisation parameter $\sigma_{8}=0.9$. Finally, we obtain the socalled "simulated observed map", $D$, that contains the three previous astrophysical components. It is obtained from $D=$ $\sqrt{\alpha} \mathrm{CMB}+\sqrt{1-\alpha} \mathrm{CS}+\mathrm{KSZ}$, where $\mathrm{CMB}, \mathrm{KSZ}$ and $\mathrm{CS}$ are respectively the $\mathrm{CMB}$, the kinetic $\mathrm{SZ}$ and the cosmic string simulated maps. $\alpha=0.82$ is a constant derived by (Bouchet et al. 2002). All the simulated maps have $500 \times 500$ pixels with a resolution of 1.5 arcmin per pixel. Apart from the dominant inflationary component which is Gaussian, all the other contributions are non-Gaussian. However, KSZ and CS have different characteristics as the KSZ and CS induce respectively spherical-like and string-like structures in the CMB. In order to illustrate how difficult the task of detecting and separating different non-Gaussian signatures is, we show in Fig. 1 a set of simulated maps. Primary CMB, kinetic SZ and cosmic string maps are shown respectively in Fig. 1 top left, top right and 


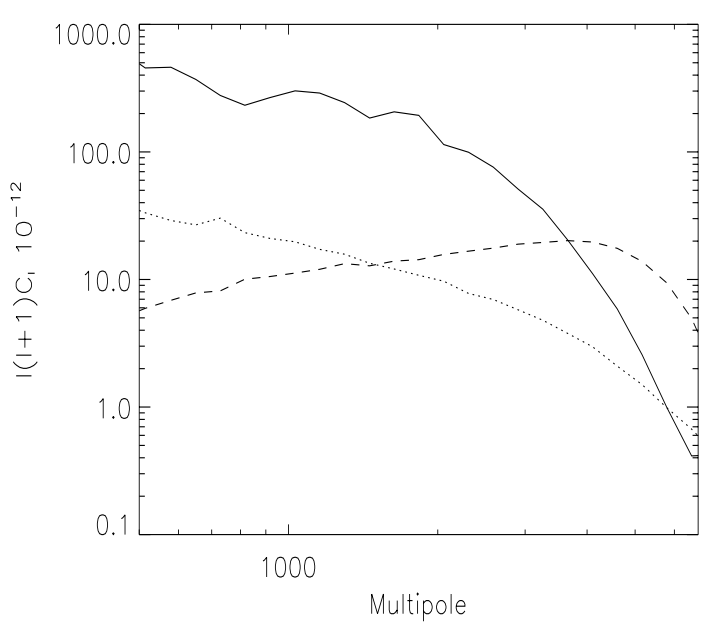

Fig. 2. Power spectra of the different contributions used in this study. The solid line represents the primary CMB anisotropies from an inflationary model (the fluctuations are Gaussian distributed). The dashed line is for the kinetic SZ effect from galaxy clusters. The dotted line stands for the power spectrum of the allowed contribution from cosmic strings, i.e. with the parameter $\alpha=0.82$.

bottom left. The "simulated observed map", containing the three previous components, is displayed in Fig. 1 bottom right. It can easily be seen from Fig. 2, which displays the power spectra of the different components, that the primary CMB anisotropies dominate all the signals except at very high multipoles (very small angular scales).

\section{Analysis and results}

\subsection{Sky component multi-scale analysis.}

On each of the three sets of maps (i.e. CMB, CS and KSZ), we have first run the following multi-scale methods:

- à trous wavelet transform;

- bi-orthogonal wavelet transform, using the standard 7/9 filter (Antonini et al. 1992);

- local ridgelet transform with a block size equal to 16 pixels;

- local ridgelet transform with a block size equal to 32 pixels;

- curvelet transform with a block size equal to 16 pixels.

This processing has been repeated on $100 \mathrm{CMB}$ and $100 \mathrm{KSZ}$ simulated maps and on the three available CS maps. In order to estimate the sensitivity of each transform to the different types of non-Gaussian signatures associated with the different components individually (sharp edges for CS and point sources for KSZ), we have calculated the mean excess kurtosis (fourth moment) and its standard deviation on each individual resolution level. In this analysis, we do not present the skewness (third moment) since we do not expect the KSZ signal to be "skewed" (Da Silva 2002). The comparison of the transforms on the basis of this non-Gaussian estimator in our case would be meaningless.

For example, the à trous wavelet transform applied to the CMB produces 100 individual values for the excess of kurtosis per scale (resolution level). We note $K_{a}(i, b)$ the excess kurtosis of the $i$ th simulated CMB map $(i=1 \ldots 100)$ at the band $b$.
Table 1. Table of the maximum value among all bands of the mean excess kurtosis $\bar{K}_{a}$ (Col. 1), its associated scale (Col. 3) and the standard deviation $\sigma_{K}$ (Col. 2) for the primary CMB anisotropies (inflationary model) only. Columns 4 and 5 give the maximum value for $\bar{K}_{a}(b) / \sigma_{K_{\mathrm{G}}}$ and the associated scale

\begin{tabular}{lccc|cc}
\hline \hline Multi-scale method & $\bar{K}_{a}$ & $\sigma_{K}$ & Scale & $\frac{\bar{K}_{a}}{\sigma_{K_{\mathrm{G}}}}$ & Scale \\
\hline Bi-orthogonal wavelet & 0.006 & 0.098 & 3,2 & 0.262 & 1,3 \\
à trous WT & -0.04 & 0.198 & 5 & 0.20 & 3 \\
Local ridgelets $(B=16)$ & 0.009 & 0.028 & 1 & 0.33 & 1 \\
Local ridgelets $(B=32)$ & 0.061 & 0.030 & 1 & 1.976 & 1 \\
Curvelets $(B=16)$ & 0.271 & 0.183 & 5,3 & 3.540 & 4,1 \\
\hline
\end{tabular}

Table 2. Table of the maximum value among all bands of the mean excess kurtosis $\bar{K}_{a}$ (Col. 1), its associated scale (Col. 3) and the standard deviation $\sigma_{K}$ (Col. 2) for the kinetic SZ fluctuations only. Columns 4 and 5 give the maximum value for $\bar{K}_{a}(b) / \sigma_{K_{\mathrm{G}}}$ and the associated scale.

\begin{tabular}{lccc|cc}
\hline \hline Multi-scale method & $\bar{K}_{a}$ & $\sigma_{K}$ & Scale & $\frac{\bar{K}_{a}}{\sigma_{K_{\mathrm{G}}}}$ & Scale \\
\hline Bi-orthogonal wavelet & 46.673 & 35.26 & 1,1 & 2081.41 & 1,3 \\
à trous WT & 80.380 & 55.898 & 1 & 4354.48 & 1 \\
Local ridgelets $(B=16)$ & 18.961 & 8.886 & 2 & 397.85 & 2 \\
Local ridgelets $(B=32)$ & 14.715 & 8.446 & 3 & 229.01 & 2 \\
Curvelets $(B=16)$ & 12.360 & 9.475 & 3,4 & 351.80 & 1,1 \\
\hline
\end{tabular}

For the à trous wavelet transform and the ridgelet transform, we have $b=j$, where $j$ is the scale $(j=1 \ldots J)$ and $J$ is number of scales of the wavelet transform (we used $J=5$ in our experiments). For a bi-orthogonal wavelet transform, we have three bands per scale $(b=1 \ldots 3 J)$, and $b$ can also be represented by the two indices $(j, d), d(d=1 \ldots 3)$ standing respectively for the horizontal, vertical or diagonal directions. The same holds for the curvelet transform where $b$ can be represented by the two indices $(j, r)$ where $r$ is the scale index in the ridgelet transform.

We derive from the ensemble of $K_{a}$ obtained on the CMB maps the mean kurtosis value $\bar{K}_{a}(b)$ and the standard deviation $\sigma_{K}(b)$. Table 1 gives the maximum value of $\bar{K}_{a}(b)$ (among all bands), its associated scale and the standard deviation (Cols. 1$3)$. It also gives the maximum value for the ratio $\bar{K}_{a}(b) / \sigma_{K_{\mathrm{G}}}$, where $\sigma_{K_{\mathrm{G}}}$ is the standard deviation of the kurtosis in the Gaussian set of maps, as well as the associated scale (Cols. 4 and 5). The same treatment is applied on the KSZ and the CS components. The results are respectively given in Tables 2 and 3 .

As it was expected, the à trous algorithm is better suited to detect isotropic features such as the "quasi-spherical" KSZ anisotropies due to galaxy clusters. Indeed, we note from Table 2 that the multi-scale excess kurtosis for KSZ is largest when using the à trous algorithm. Unexpectedly though, the multi-scale excess kurtosis for CS (Table 3) is largest when using the bi-orthogonal wavelet transform whereas the excess kurtosis from the ridgelet and the curvelet transforms show that both transforms seem not that well adapted to test the CS nonGaussian signatures. An explanation for this behaviour is that 
Table 3. Table of the maximum value among all bands of the mean excess kurtosis $\bar{K}_{a}$ (Col. 1), its associated scale (Col. 3) and the standard deviation $\sigma_{K}$ (Col. 2) for the cosmic strings only. Columns 4 and 5 give the maximum value for $\bar{K}_{a}(b) / \sigma_{K_{\mathrm{G}}}$ and the associated scale. Note that only 3 maps are analysed in this case.

\begin{tabular}{lccc|cc}
\hline \hline Multi-scale method & $\bar{K}_{a}$ & $\sigma_{K}$ & Scale & $\frac{\bar{K}_{a}}{\sigma_{K_{\mathrm{G}}}}$ & Scale \\
\hline Bi-orthogonal wavelet & 51.36 & 1.90 & 1,2 & 2059.74 & 1,2 \\
à trous WT & 35.76 & 7.99 & 1 & 1937.78 & 1 \\
Local ridgelets $(B=16)$ & 6.98 & 0.95 & 1 & 243.02 & 1 \\
Local ridgelets $(B=32)$ & 3.69 & 0.46 & 1 & 117.67 & 1 \\
Curvelets $(B=16)$ & 10.43 & 1.40 & 1,1 & 609.06 & 1,1 \\
\hline
\end{tabular}

the CS (see Fig. 1) presents not only elongated structures and sharp edges, but also a large number of spots and sphericallike structures along the edges and at the intersection of edges, which contribute significantly to the non-Gaussian character. These spots are very well detected by the wavelets.

\subsection{Testing the sensitivities to Gaussian + non-Gaussian signals}

In this section, we study the relative sensitivity of the different multi-scale transforms to the two families of different non-Gaussian characters when the signals are added to a dominant Gaussian noise, i.e. the primary CMB. We use three datasets $D^{(1)}, D^{(2)}$ and $D^{(3)}$. We have created 300 simulated maps by adding the $100 \mathrm{CMB}$ realisations to the KSZ $\left(D_{i}^{(1)}=\mathrm{CMB}_{i}+\mathrm{KSZ}\right.$, and $\left.i=1 \ldots 100\right)$, to the CS $\left(D_{i}^{(2)}=\right.$ $\left.\sqrt{\alpha} \mathrm{CMB}_{i}+\sqrt{1-\alpha} \mathrm{CS}\right)$, and to both the KSZ and CS components $\left(D_{i}^{(3)}=\sqrt{\alpha} \mathrm{CMB}_{i}+\sqrt{1-\alpha} \mathrm{CS}+\mathrm{KSZ}\right)$. Then we apply our five multi-scale transforms to these 300 maps. As in the previous section, we have calculated for each band $b$ of each transform and for each dataset $D^{(l)}(l=1,2,3)$ the mean kurtosis value $\bar{K}_{D^{(l)}}(b)$ and the standard deviation $\sigma_{K_{D^{(l)}}, b}$. In order to calibrate and compare the departures from a Gaussian distribution, we have simulated for each image $D_{i}^{(l)}$ a Gaussian Random Field $G_{i}^{(l)}$ which has the same power spectrum as $D_{i}^{(l)}$. This allows us to calculate a normalised mean kurtosis given by:

$\mathcal{K}_{l}(j)=\frac{\bar{K}_{D^{(l)}}(b)-\bar{K}_{G^{(l)}}(b)}{\sigma_{K_{G^{(l)}}, b}}$.

We give in Tables 4-6 the results in terms of the maximum values for illustration. Complete tables can be found in Appendix A for the three datasets $D^{(1)}, D^{(2)}$ and $D^{(3)}$. The first three columns represent maximum $\bar{K}_{a}$, standard deviation and associated scale. The last two columns give the maximum normalised mean kurtosis and associated scale.

From Tables 4-6, we note that:

- Whatever the transform, it is at the first resolution level that the non-Gaussian character is the best detected.

- The third band of the bi-orthogonal wavelet transform (i.e. diagonal details of the first wavelet scale) is generally the best for detecting non-Gaussian signatures in the CMB,
Table 4. Table of maximum mean excess kurtosis $\bar{K}_{a}$, and associated standard deviation $\sigma_{K}$ and scale (first three columns). The last two columns give the maximum normalised mean kurtosis $\mathcal{K}$ and associated scale for $D^{(1)}=\mathrm{CMB}+\mathrm{KSZ}$.

\begin{tabular}{lccc|cc}
\hline \hline Multi-scale method & $\bar{K}_{a}$ & $\sigma_{K}$ & Scale & $\mathcal{K}$ & Scale \\
\hline Bi-orthogonal wavelet & 22.94 & 0.098 & 1,3 & 1106.58 & 1,3 \\
à trous WT & 0.73 & 0.06 & 1 & 65.79 & 1 \\
Local ridgelets $(B=16)$ & 0.013 & 0.029 & 1 & 0.124 & 1 \\
Local ridgelets $(B=32)$ & 0.062 & 0.030 & 1 & 0.114 & 1 \\
Curvelets $(B=16)$ & 0.276 & 0.109 & 4,3 & 10.12 & 1,1 \\
\hline
\end{tabular}

Table 5. Table of maximum mean excess kurtosis $\bar{K}_{a}$, and associated standard deviation $\sigma_{K}$ and scale (first three columns). The last two columns give the maximum normalised mean kurtosis $\mathcal{K}$ and associated scale for $D^{(2)}=\mathrm{CMB}+\mathrm{CS}$.

\begin{tabular}{lccc|cc}
\hline \hline Multi-scale method & $\bar{K}_{a}$ & $\sigma_{K}$ & Scale & $\mathcal{K}$ & Scale \\
\hline Bi-orthogonal wavelet & 37.96 & 0.14 & 1,3 & 1813.61 & 1,3 \\
à trous WT & 5.74 & 0.15 & 1 & 424.15 & 1 \\
Local ridgelets $(B=16)$ & 0.174 & 0.033 & 1 & 5.68 & 1 \\
Local ridgelets $(B=32)$ & 0.152 & 0.031 & 1 & 2.84 & 1 \\
Curvelets $(B=16)$ & 2.22 & 0.055 & 1,1 & 198.62 & 1,1 \\
\hline
\end{tabular}

Table 6. Table of maximum mean excess kurtosis $\bar{K}_{a}$, and associated standard deviation $\sigma_{K}$ and scale (first three columns). The last two columns give the maximum normalised mean kurtosis $\mathcal{K}$ and associated scale for $D^{(3)}=\mathrm{CMB}+\mathrm{KSZ}+\mathrm{CS}$.

\begin{tabular}{lccc|cc}
\hline \hline Multi-scale method & $\bar{K}_{a}$ & $\sigma_{K}$ & Scale & $\mathcal{K}$ & Scale \\
\hline Bi-orthogonal wavelet & 26.22 & 0.53 & 1,2 & 1040.54 & 1,2 \\
à trous WT & 5.20 & 0.12 & 1 & 392.25 & 1 \\
Local ridgelets $(B=16)$ & 0.17 & 0.032 & 1 & 5.88 & 1 \\
Local ridgelets $(B=32)$ & 0.149 & 0.030 & 1 & 2.99 & 1 \\
Curvelets $(B=16)$ & 1.79 & 0.046 & 1,1 & 165.68 & 1,1 \\
\hline
\end{tabular}

even if the structures are isotropic and spherical-like (see previous section).

- The most important result is that the normalised mean kurtosis of the ridgelets is compatible with 0 in the case $\mathrm{CMB}+\mathrm{KSZ}$ whereas it is non-zero in the case $\mathrm{CMB}+\mathrm{CS}$. Moreover, it conserves the same value in the case $\mathrm{CMB}+\mathrm{SZ}+\mathrm{CS}$. For the normalised mean kurtosis of the curvelets there is a non-zero value for the $\mathrm{CMB}+\mathrm{KSZ}$ signal but it is almost 20 times smaller than in the cases $\mathrm{CMB}+\mathrm{CS}$ and $\mathrm{CMB}+\mathrm{SZ}+\mathrm{CS}$.

The first remark is related to the relative contributions of the different astrophysical components. The primary CMB dominates over the other components except at the first decomposition (or wavelet) scale as can be seen from Fig. 2. At this scale corresponding typically to 3 arcmin, the contribution from the SZ effect and the CS is of the order of, or dominates, the primary CMB. The non-Gaussian signatures introduced by both KSZ and CS are thus easier to detect at this scale. 
It is worth noting however, that the non-Gaussian character remains detectable at larger scales (second and third decomposition scales).

The second point was already mentioned in Aghanim \& Forni (1999). In the previous section we have shown that the spherical-like sources of non-Gaussianity were better detected by the à trous transform when they are not mixed with a dominant Gaussian signal (the CMB here). This property is not conserved when the quasi-spherical non-Gaussianities such as the KSZ effect are added to the primary CMB. The reason why the so-called diagonal details are more sensitive than the other tests is the following: In the Fourier domain, we note $R(u, v)=\frac{S(u, v)}{C(u, v)}$, the Signal-to-Noise ratio (SNR), where $S$ is the power spectrum of the non-Gaussian signal and $C$ is the power spectrum of the primary CMB (Gaussian signal in our case). As the CMB power spectrum is isotropic, we have $R(u, v)=\frac{S(u, v)}{C\left(\sqrt{u^{2}+v^{2}}\right)}$. When $\rho=\sqrt{u^{2}+v^{2}}$ increases, the SNR increases, which explains why the first wavelet scale presents the highest sensitivity to the non-Gaussian features. Additionally, it becomes clear that $R(u, v)$ is higher for $u=v=N / 2$ rather than for $u=0$ and $v=N / 2$ or $u=N / 2$ and $v=0$, which implies that the diagonal band (or details) is more sensitive than horizontal and vertical bands, and confirms the results described in Aghanim \& Forni (1999).

The third point is undoubtedly the most important result of this comparison. It shows that the normalised mean kurtosis of the ridgelets is able to highlight the non-Gaussian character of the CS buried in the CMB Gaussian signal with a mixing ratio of 0.18 (in power). The normalised mean kurtosis of the ridgelets are sensitive only to the string-like non-Gaussianities. The numbers are the same for the $\mathrm{CMB}+\mathrm{CS}$ and $\mathrm{CMB}+\mathrm{SZ}+\mathrm{CS}$ cases and there is no detection in the $\mathrm{CMB}+\mathrm{KSZ}$. A similar behaviour is also noticeable for the normalised mean kurtosis of the curvelets. In this case, there is a detection of the nonGaussian character associated with the KSZ effect which is one order of magnitude smaller than in the case of $\mathrm{CMB}+\mathrm{SZ}+\mathrm{CS}$. These behaviours discussed at the first decomposition scale are true for larger scales $(j=2)$.

From this last remark, we already see that we have found a family of multi-resolution transforms, namely anisotropic based-systems (ridgelets and curvelets) that are sensitive to a unique family of non-Gaussian signatures, namely the stringlike structures. In the following section, we propose a strategy based on our results to discriminate between spherical-like and string-like contributions to the non-Gaussian signatures.

\subsection{Discriminating between the non-Gaussian signatures}

If a non-Gaussian signature is detected in the CMB by a given method, it can be due to calibration problems or to several astrophysical components such as KSZ or CS. Therefore, an excess kurtosis may be very difficult to interpret in terms of its origin. While some limits can be put on the excess due to instrumental or calibration problems, the discrimination between astrophysical components is not obvious.
Table 7. Product of the normalised excess kurtosis.

\begin{tabular}{lcccc}
\hline \hline Kurtosis product & bands & CMB+CS & CMB+SZ & CMB+SZ+CS \\
\hline $\mathcal{K}_{\mathrm{AT}} * \mathcal{K}_{\mathrm{CUR}}$ & $1-1,1$ & 84248. & 665.8 & 64990.1 \\
& $1-1,2$ & 2225.8 & 0.702 & 2163.28 \\
& $2-2,2$ & 57.74 & 0.013 & 66.024 \\
& $2-2,3$ & 7.97 & 0.093 & 8.58 \\
$\mathcal{K}_{\mathrm{AT}} * \mathcal{K}_{R 16}$ & $1-1$ & 2410.19 & 8.21 & 2307.2 \\
& $2-2$ & 8.50 & 0.082 & 9.465 \\
$\mathcal{K}_{\mathrm{AT}} * \mathcal{K}_{R 32}$ & $1-1$ & 1204.6 & 3.978 & 1172.0 \\
& $2-2$ & 4.547 & 0.139 & 4.692 \\
& $3-3$ & 0.017 & 0.001 & 0.004 \\
\hline
\end{tabular}

We investigate in this section a method by which the use of several multi-scale transforms helps us in understanding the nature of the detected non-Gaussian features. In particular, we focus on the possibility to discriminate the case "CMB+SZ" from the case "CMB+SZ+CS". From Tables 4-6, we can easily see that the wavelet transforms are sensitive to both the KSZ and the CS, while the ridgelet transform and the curvelet transform are not or are less sensitive to the KSZ. However, the numbers we obtain for the normalised mean excess kurtosis are of the order of a few in the best cases. It is thus important to find a better estimator of the non-Gaussian signatures. We require an estimator that enhances the signal to noise ratio. The product of the normalised kurtosis obtained by two different transforms is a promising approach for our problem. We note $\mathcal{K}_{\mathrm{AT}}, \mathcal{K}_{\mathrm{CUR}}, \mathcal{K}_{R 16}, \mathcal{K}_{R 32}$ the normalised kurtosis using the à trous wavelet transform, the curvelet transform, the ridgelet transform with a block size equal to 16 and the ridgelet transform with a block size equal to 32 respectively. Table 7 gives such products. The first column indicates the two band numbers used, the last three columns give the value of the product for the cases $\mathrm{CMB}+\mathrm{CS}, \mathrm{CMB}+\mathrm{SZ}$ and $\mathrm{CMB}+\mathrm{SZ}+\mathrm{CS}$ respectively. We have given illustrative values. The whole set of values can be found in Appendix A (Table 11).

A significant detection of non-Gaussianity by the product of wavelet-like and curvelet-like transforms must rely on comparable bands. More specifically, the curvelet bands noted $(j, r)$ for which $j$ and $r$ are too different, are meaningless by construction. Moreover, for the largest decomposition levels, the number of coefficients are small and the results are thus more sensitive to sample variance.

We see from these values that the product of the normalised kurtosis of the wavelet transform (here the à trous transform for illustration ${ }^{3}$ ) by normalised kurtosis of the curvelet transform clearly discriminates between the case $\mathrm{CMB}+\mathrm{SZ}$ and $\mathrm{CMB}+\mathrm{SZ}+\mathrm{CS}$. This is true up to the second decomposition scale.

\footnotetext{
3 Similar results are obtained for the OWT transform but in this case there are 3 bands per scale and many more combinations and products are possible. The associated numbers can be obtained upon request
} 


\section{Discussion}

We tackle the problem of the separation between different sources of non-Gaussian signatures. We are not yet able to identify the exact contribution of the different effects but rather we detect that there are two families of non-Gaussian features.

In "real-life" we will of course be a priori unable to separate perfectly the two contributions, KSZ and CS, from the primary CMB. Tables such as Table 7 will therefore only contain the numbers associated with the case $\mathrm{CMB}+\mathrm{CS}+\mathrm{KSZ}$ and no relative detection will be made. However, the simulations allow us to calibrate the method. We show (Table 7) that there can be orders of magnitudes of difference in terms of the products of normalised kurtosis between cases where CS are present and cases where they are not. We can therefore conclude from our study that whenever the product of the normalised kurtosis from the wavelets and from the curvelets (or ridgelets) are of the order of a few this indicates that string-like non-Gaussian features are present in the total signal. A larger number would allow us to fully characterise the non-Gaussian properties of the signals and to calibrate the method.

At this stage, the precise effects of the instrumental noise and of the beam convolution are not taken into account and performing such an analysis is beyond the scope of our study. However, the non-Gaussian signals are detected not only at the first decomposition scale but also at larger scales (up to $j=3$ ). The beam convolution is likely to affect the first and second scale but present and future instruments (Planck, ACT, SPT, ACBAR) will have better angular resolution and the beam effects are likely to be less important. As for the noise, if we assume it Gaussian (which is the case in most studies) it will make the non-Gaussian signatures more difficult to detect by reducing the overall signal to noise ratio. However, the numbers presented in Table 7 show that the detection of CS is quite significant and we do not expect it to vanish if white noise is added unless the noise has similar features as a CS. More generally, in the case of any systematic effect exhibiting anisotropic structures our method will detect them without a priori being able to disentangle their origin from a cosmological origin like the CS.

In this analysis, we present only the excess kurtosis as an estimator of non-Gaussianity. We perform a comparison of the multi-scale transforms and of their sensitivity to the signals on the basis of the non-Gaussian character to highlight different contributors. Since we do not expect the KSZ signal to be "skewed", a comparison based on the skewness (third moment) would be meaningless. As expected, our simulations have shown that the skewness is indeed not as sensitive as the kurtosis.

\section{Conclusion}

In the present study, we have tested the relative sensitivities of the commonly used multi-scale transforms to the detection of non-Gaussian signatures in CMB maps. We have used simulated maps of the primary anisotropies (assumed to be Gaussian) and we have taken into account the contributions from the kinetic SZ effect and the topological defects (cosmic strings); each of them represents one family of non-Gaussian contributors (spherical-like and string-like). The main results are:

- the bi-orthogonal wavelet transform is the most sensitive to the non-Gaussian signatures associated with cosmic strings buried in the primary Gaussian CMB signal.

- The ridgelet and curvelet transforms are well adapted to discriminate between string-like and quasi-spherical like non-Gaussian features.

In order to study the non-Gaussian signatures in the CMB and use them as a cosmological tool to probe the early universe or the cosmic structures, it is not sufficient to detect them accurately. The most important step is to be able to separate the different contributions to the signal. In this context, we clearly show that not only one method should be applied but rather a set of different robust and well understood methods.

In the present study, we use several multi-scale transforms: the isotropic wavelet transforms suited for sphericallike sources of non-Gaussianity, and a curvelet transform representing well sharp and elongated structures. Each provides an adapted non-Gaussian estimator, namely the normalised mean excess kurtosis. We show that the combination of these transforms through the product of the normalised mean excess kurtosis of isotropic wavelet transforms by normalised mean excess kurtosis of curvelet transforms highlights the presence of the cosmic strings in a mixture $\mathrm{CMB}+\mathrm{KSZ}+\mathrm{CS}$. Such a combination gives information about the nature of the non-Gaussian signals. Even though the detection power of the non-Gaussian character by the ridgelets and curvelets is not important compared to that of the wavelet, their sensitivity to a particular shape makes them a very strong discriminating tool.

This is a first step towards the separation of the statistical contributions to the CMB signal and it can help in the more general context of component separation.

Acknowledgements. The cosmic string maps were kindly provided by F. R. Bouchet. We wish to thank David Donoho for useful discussions. We are grateful to the referee for helpful comments on an earlier version.

\section{References}

Aghanim, N., \& Forni, O. 1999, A\&A, 347, 409

Aghanim, N., Górski, K. M., \& Puget, J.-L. 2001, A\&A, 374, 1

Antonini, M., Barlaud, M., Mathieu, P., \& Daubechies, I. 1992, IEEE

Transactions on Image Processing, 1, 205

Banday, A. J., Zaroubi, S., \& Górski, K. M. 2000, ApJ, 533, 575

Barreiro, R. B., \& Hobson, M. P. 2001, MNRAS, 327, 813

Bennett, C. L., Halpern, M., Hinshaw, G., et al. 2003, ApJS, 148, 1

Bernardeau, F., \& Uzan, J. 2002, Phys. Rev. D, 66, 103506

Bernardeau, F., van Waerbeke, L., \& Mellier, Y. 2003, A\&A, 397, 405

Bouchet, F. R., Bennett, D. P., \& Stebbins, A. 1988, Nature, 335, 410

Bouchet, F. R., Peter, P., Riazuelo, A., \& Sakellariadou, M. 2002, Phys. Rev. D, 65, 21301

Bromley, B. C., \& Tegmark, M. 1999, ApJ, 524, L79

Candès, E., \& Donoho, D. 1999a, Phil. Trans. R. Soc. London A, 357, 2495 
Candès, E. J., \& Donoho, D. L. 1999b, in Curve and Surface Fitting: Saint-Malo 1999, ed. A. Cohen, C. Rabut, \& L. Schumaker, (Nashville, TN: Vanderbilt University Press)

Castro, P. G. 2003, Phys. Rev. D, 67, 123001

Cayón, L., Sanz, J. L., Martínez-González, E., et al. 2001, MNRAS, 326,1243

Cooray, A. 2001, Phys. Rev. D, 64, 3514

Da Silva, A. J. C. 2002, Ph.D. Thesis, Sussex university

De Troia, G., Ade, P. A. R., Bock, J. J., et al. 2003, MNRAS, 343, 284

Donoho, D., \& Duncan, M. 2000, in Proc. Aerosense 2000, ed. H. Szu, M. Vetterli, W. Campbell, \& J. Buss, Wavelet Applications VII, 4056, 12, SPIE

Forni, O., \& Aghanim, N. 1999, A\&AS, 137, 553

Hobson, M. P., Jones, A. W., \& Lasenby, A. N. 1999, MNRAS, 309, 125

Jewell, J. 2001, ApJ, 557, 700

Komatsu, E., Kogut, A., Nolta, M. R., et al. 2003, ApJS, 148, 119

Kunz, M., Banday, A. J., Castro, P. G., Ferreira, P. G., \& Górski, K. M. 2001, ApJ, 563, L99

Mallat, S. 1998, A Wavelet Tour of Signal Processing (Academic Press)

Novikov, D., Schmalzing, J., \& Mukhanov, V. F. 2000, A\&A, 364, 17
Ostriker, J. P., \& Vishniac, E. T. 1986, ApJ, 306, L51

Phillips, N. G., \& Kogut, A. 2001, ApJ, 548, 540

Riazuelo, A., Uzan, J.-P., Lehoucq, R., \& Weeks, J. 2002, Simulating Cosmic Microwave Background maps in multi-connected spaces [astro-ph/0212223]

Rousseeuw, P., \& Croux, C. 1993, J. Amer. Stat. Asssociation, 88, 1273

Shandarin, S. F. 2002, MNRAS, 331, 865

Starck, J.-L., Candès, E., \& Donoho, D. 2002, IEEE Transactions on Image Processing, 11, 131

Starck, J.-L., Candes, E., \& Donoho, D. 2003, A\&A, 398, 785

Starck, J.-L., \& Murtagh, F. 2002, Astronomical Image and Data Analysis (Springer-Verlag)

Starck, J.-L., Murtagh, F., \& Bijaoui, A. 1998, Image Processing and Data Analysis: The Multiscale Approach (Cambridge University Press)

Sunyaev, R. A., \& Zeldovich, I. B. 1980, ARA\&A, 18, 537

Verde, L., Wang, L., Heavens, A. F., \& Kamionkowski, M. 2000, MNRAS, 313, 141

Vishniac, E. T. 1987, ApJ, 322, 597

White, M., \& Cohn, J. D. 2002, The Theory of Anisotropies in the Cosmic Microwave Background [astro-ph/0203120] 


\section{Online Material}


J.-L. Starck et al.: Detection and discriminating the cosmological non-Gaussian signatures, Online Material p 2

\section{Appendix A}

Table 8. Table of mean excess kurtosis and standard deviation for $D^{(1)}=\mathrm{CMB}+\mathrm{KSZ}$.

\begin{tabular}{|c|c|c|c|c|}
\hline Multi-scale method & Scale & $\bar{K}_{a}$ & $\sigma_{K}$ & $\mathcal{K}$ \\
\hline \multirow[t]{15}{*}{ Bi-orthogonal wavelet } & 1,1 & 6.305 & 0.188 & 275.096 \\
\hline & 1,2 & 7.205 & 0.270 & 314.839 \\
\hline & 1,3 & 22.942 & 0.098 & 1106.586 \\
\hline & 2,1 & 0.068 & 0.058 & 1.788 \\
\hline & 2,2 & 0.057 & 0.056 & 1.416 \\
\hline & 2,3 & 1.782 & 0.151 & 47.186 \\
\hline & 3,1 & 0.013 & 0.088 & 0.134 \\
\hline & 3,2 & 0.009 & 0.098 & 0.040 \\
\hline & 3,3 & -0.002 & 0.082 & 0.090 \\
\hline & 4,1 & -0.038 & 0.180 & 0.013 \\
\hline & 4,2 & -0.014 & 0.184 & 0.008 \\
\hline & 4,3 & -0.005 & 0.175 & 0.064 \\
\hline & 5,1 & -0.088 & 0.323 & 0.011 \\
\hline & 5,2 & -0.111 & 0.466 & 0.023 \\
\hline & 5,3 & -0.042 & 0.530 & 0.023 \\
\hline \multirow[t]{5}{*}{ à trous WT } & 1 & 0.731 & 0.060 & 65.793 \\
\hline & 2 & 0.018 & 0.031 & 1.209 \\
\hline & 3 & -0.006 & 0.048 & 0.029 \\
\hline & 4 & -0.017 & 0.101 & 0.095 \\
\hline & 5 & -0.042 & 0.198 & 0.060 \\
\hline \multirow[t]{2}{*}{ Local ridgelets $(B=16)$} & 1 & 0.013 & 0.029 & 0.124 \\
\hline & 2 & 0.008 & 0.048 & 0.067 \\
\hline \multirow[t]{3}{*}{ Local ridgelets $(B=32)$} & 1 & 0.062 & 0.030 & 0.060 \\
\hline & 2 & 0.058 & 0.044 & 0.114 \\
\hline & 3 & 0.040 & 0.106 & 0.0394 \\
\hline \multirow[t]{18}{*}{ Curvelets $(B=16)$} & 1,1 & 0.117 & 0.016 & 10.120 \\
\hline & 1,2 & 0.020 & 0.029 & 0.010 \\
\hline & 1,3 & 0.017 & 0.094 & 0.077 \\
\hline & 2,1 & 0.064 & 0.023 & 0.207 \\
\hline & 2,2 & 0.065 & 0.038 & 0.019 \\
\hline & 2,3 & 0.058 & 0.056 & 0.029 \\
\hline & 2,4 & 0.05 & 0.175 & 0.072 \\
\hline & 3,1 & 0.060 & 0.034 & 0.084 \\
\hline & 3,2 & 0.060 & 0.045 & 0.082 \\
\hline & 3,3 & 0.058 & 0.073 & 0.016 \\
\hline & 3,4 & 0.028 & 0.196 & 0.251 \\
\hline & 4,1 & 0.257 & 0.073 & 0.009 \\
\hline & 4,2 & 0.261 & 0.083 & 0.038 \\
\hline & 4,3 & 0.276 & 0.109 & 0.080 \\
\hline & 4,4 & 0.260 & 0.250 & 0.051 \\
\hline & 5,1 & 0.262 & 0.146 & 0.016 \\
\hline & 5,2 & 0.272 & 0.149 & 0.017 \\
\hline & 5,3 & 0.274 & 0.187 & 0.041 \\
\hline
\end{tabular}

Table 9. Table of mean excess kurtosis and standard deviation for $D^{(2)}=\mathrm{CMB}+\mathrm{CS}$.

\begin{tabular}{|c|c|c|c|c|}
\hline Multi-scale method & Scale & $\bar{K}_{a}$ & $\sigma_{K}$ & $\mathcal{K}$ \\
\hline \multirow[t]{15}{*}{ Bi-orthogonal wavelet } & 1,1 & 18.369 & 0.292 & 756.618 \\
\hline & 1,2 & 37.797 & 0.763 & 1550.101 \\
\hline & 1,3 & 37.960 & 0.139 & 1813.615 \\
\hline & 2,1 & 1.662 & 0.179 & 36.929 \\
\hline & 2,2 & 1.823 & 0.204 & 35.329 \\
\hline & 2,3 & 10.051 & 0.380 & 261.331 \\
\hline & 3,1 & 0.082 & 0.085 & 0.820 \\
\hline & 3,2 & 0.098 & 0.105 & 1.052 \\
\hline & 3,3 & 0.257 & 0.136 & 2.683 \\
\hline & 4,1 & -0.023 & 0.181 & 0.064 \\
\hline & 4,2 & 0.034 & 0.194 & 0.476 \\
\hline & 4,3 & -0.001 & 0.165 & 0.032 \\
\hline & 4,1 & -0.060 & 0.354 & 0.097 \\
\hline & 4,2 & -0.111 & 0.463 & 0.059 \\
\hline & 4,3 & -0.037 & 0.479 & 0.086 \\
\hline \multirow[t]{5}{*}{ à trous WT } & 1 & 5.744 & 0.153 & 424.159 \\
\hline & 2 & 0.250 & 0.049 & 11.003 \\
\hline & 3 & 0.030 & 0.055 & 0.688 \\
\hline & 4 & -0.004 & 0.097 & 0.233 \\
\hline & 5 & -0.032 & 0.191 & 0.271 \\
\hline \multirow[t]{2}{*}{ Local ridgelets $(B=16)$} & 1 & 0.174 & 0.034 & 5.682 \\
\hline & 2 & 0.055 & 0.049 & 0.773 \\
\hline \multirow[t]{3}{*}{ Local ridgelets $(B=32)$} & 1 & 0.153 & 0.031 & 2.840 \\
\hline & 2 & 0.082 & 0.047 & 0.413 \\
\hline & 3 & 0.066 & 0.113 & 0.024 \\
\hline \multirow[t]{18}{*}{ Curvelets $(B=16)$} & 1,1 & 2.222 & 0.055 & 198.625 \\
\hline & 1,2 & 0.157 & 0.044 & 5.248 \\
\hline & 1,3 & 0.059 & 0.102 & 0.725 \\
\hline & 2,1 & 0.206 & 0.028 & 7.591 \\
\hline & 2,2 & 0.100 & 0.042 & 1.108 \\
\hline & 2,3 & 0.072 & 0.062 & 0.265 \\
\hline & 2,4 & 0.053 & 0.172 & 0.197 \\
\hline & 3,1 & 0.080 & 0.033 & 0.820 \\
\hline & 3,2 & 0.075 & 0.048 & 0.565 \\
\hline & 3,3 & 0.067 & 0.082 & 0.127 \\
\hline & 3,4 & 0.007 & 0.180 & 0.123 \\
\hline & 4,1 & 0.258 & 0.067 & 0.115 \\
\hline & 4,2 & 0.261 & 0.073 & 0.080 \\
\hline & 4,3 & 0.278 & 0.108 & 0.153 \\
\hline & 4,4 & 0.278 & 0.269 & 0.092 \\
\hline & 5,1 & 0.271 & 0.132 & 0.052 \\
\hline & 5,2 & 0.285 & 0.142 & 0.034 \\
\hline & 5,3 & 0.290 & 0.186 & 0.023 \\
\hline
\end{tabular}


J.-L. Starck et al.: Detection and discriminating the cosmological non-Gaussian signatures, Online Material p 3

Table 10. Table of mean excess kurtosis and standard deviation for $D^{(3)}=\mathrm{CMB}+\mathrm{KSZ}+\mathrm{CS}$.

\begin{tabular}{|c|c|c|c|c|}
\hline Multi-scale method & Scale & $\bar{K}_{a}$ & $\sigma_{K}$ & $\mathcal{K}$ \\
\hline \multirow{15}{*}{ Bi-orthogonal wavelet } & 1,1 & 12.264 & 0.194 & 491.426 \\
\hline & 1,2 & 26.225 & 0.532 & 1040.543 \\
\hline & 1,3 & 20.180 & 0.069 & 933.862 \\
\hline & 2,1 & 1.503 & 0.160 & 34.154 \\
\hline & 2,2 & 1.697 & 0.187 & 33.660 \\
\hline & 2,3 & 7.477 & 0.291 & 196.930 \\
\hline & 3,1 & 0.092 & 0.089 & 0.922 \\
\hline & 3,2 & 0.095 & 0.103 & 1.027 \\
\hline & 3,3 & 0.258 & 0.130 & 2.749 \\
\hline & 4,1 & -0.025 & 0.182 & 0.073 \\
\hline & 4,2 & 0.024 & 0.195 & 0.425 \\
\hline & 4,3 & -0.000 & 0.166 & 0.035 \\
\hline & 5,1 & -0.072 & 0.341 & 0.072 \\
\hline & 5,2 & -0.110 & 0.462 & 0.062 \\
\hline & 5,3 & -0.041 & 0.470 & 0.076 \\
\hline \multirow[t]{5}{*}{ à trous WT } & 1 & 5.206 & 0.127 & 392.254 \\
\hline & 2 & 0.260 & 0.051 & 11.972 \\
\hline & 3 & 0.032 & 0.055 & 0.747 \\
\hline & 4 & -0.004 & 0.098 & 0.233 \\
\hline & 5 & -0.033 & 0.187 & 0.280 \\
\hline \multirow[t]{2}{*}{ Local ridgelets $(B=16)$} & 1 & 0.171 & 0.032 & 5.882 \\
\hline & 2 & 0.056 & 0.051 & 0.791 \\
\hline \multirow[t]{3}{*}{ Local ridgelets $(B=32)$} & 1 & 0.150 & 0.031 & 2.988 \\
\hline & 2 & 0.080 & 0.047 & 0.392 \\
\hline & 3 & 0.063 & 0.114 & 0.006 \\
\hline \multirow[t]{17}{*}{ Curvelets $(B=16)$} & 1,2 & 0.155 & 0.044 & 5.515 \\
\hline & 1,3 & 0.058 & 0.099 & 0.717 \\
\hline & 2,1 & 0.197 & 0.029 & 7.426 \\
\hline & 2,2 & 0.099 & 0.041 & 1.091 \\
\hline & 2,3 & 0.071 & 0.061 & 0.254 \\
\hline & 2,4 & 0.049 & 0.173 & 0.172 \\
\hline & 3,1 & 0.079 & 0.033 & 0.803 \\
\hline & 3,2 & 0.073 & 0.046 & 0.519 \\
\hline & 3,3 & 0.067 & 0.081 & 0.128 \\
\hline & 3,4 & 0.004 & 0.178 & 0.135 \\
\hline & 4,1 & 0.258 & 0.068 & 0.121 \\
\hline & 4,2 & 0.258 & 0.075 & 0.047 \\
\hline & 4,3 & 0.272 & 0.105 & 0.108 \\
\hline & 4,4 & 0.276 & 0.261 & 0.076 \\
\hline & 5,1 & 0.270 & 0.133 & 0.053 \\
\hline & 5,2 & 0.284 & 0.144 & 0.028 \\
\hline & 5,3 & 0.287 & 0.186 & 0.016 \\
\hline
\end{tabular}

Table 11. Kurtosis product.

\begin{tabular}{ccccc}
\hline \hline Kurtosis product & band & CMB+CS & CMB+SZ & CMB+SZ+CS \\
\hline $\mathcal{K}_{\mathrm{AT}} * \mathcal{K}_{\text {CUR }}$ & $1-1,1$ & 84248.508 & 665.882 & 64990.101 \\
& $1-1,2$ & 2225.881 & 0.702 & 2163.283 \\
$1-1,3$ & 307.422 & 5.079 & 281.125 \\
$2-2,1$ & 2185.421 & 12.246 & 1983.507 \\
& $2-2,2$ & 57.740 & 0.013 & 66.024 \\
$2-2,3$ & 7.975 & 0.093 & 8.580 \\
$2-2,4$ & 83.517 & 0.251 & 88.906 \\
$3-3,1$ & 136.694 & 0.295 & 123.816 \\
& $3-3,2$ & 3.612 & 0.000 & 4.121 \\
$3-3,3$ & 0.499 & 0.002 & 0.536 \\
$3-3,4$ & 5.224 & 0.006 & 5.550 \\
$4-4,1$ & 46.342 & 0.966 & 38.613 \\
$4-4,2$ & 1.224 & 0.001 & 1.285 \\
$4-4,3$ & 0.169 & 0.007 & 0.167 \\
$4-4,4$ & 1.771 & 0.020 & 1.731 \\
$5-5,1$ & 53.779 & 0.610 & 46.327 \\
$5-5,2$ & 1.421 & 0.001 & 1.542 \\
$5-5,3$ & 0.196 & 0.005 & 0.200 \\
\hline
\end{tabular}

